\title{
Local Time-Stepping Algorithm for Solving Probability Density Function Turbulence Model Equations
}

\author{
M. Muradoglu* and S. B. Pope ${ }^{\dagger}$ \\ Cornell University, Ithaca, New York 14853
}

\begin{abstract}
A local time-stepping algorithm has been developed to improve the numerical efficiency of Lagrangian particlebased Monte Carlo methods for obtaining the steady-state solutions of the modeled probability density function equations of turbulent reacting flows. On each step in the pseudo-time-marching algorithm, the properties of each particle are advanced by a time step, the magnitude of which depends on the particle's spatial location. This algorithm has been incorporated into the consistent hybrid finite volume/particle method. The performance of the local time-stepping method is evaluated in terms of numerical efficiency and accuracy through application to a nonreacting bluff-body flow. For this test case, it is found that local time stepping can accelerate the global convergence of the hybrid method by as much as an order of magnitude, depending on the grid stretching. Additionally, local time stepping is found to improve significantly the robustness of the hybrid method mainly due to the accelerated convection of error waves out of the computational domain. The method is very simple to implement, and the small increase in CPU time per step (typically $3 \%$ ) is a negligible penalty compared to the substantial reduction in the number of time steps required to reach convergence.
\end{abstract}

\section{Nomenclature}

$C_{u}=$ Courant number based on particle velocity

$C_{\Omega}=$ Courant number based on turbulent frequency

$D_{b}=$ bluff-body diameter

$\mathcal{F}=$ mass-density function for uniform time stepping

$\hat{\mathcal{F}}=$ mass-density function for the local time stepping

$\tilde{f}=$ mass-weighted probability density function

$M^{2}=$ total number of grid cells

$m=$ particle mass

$q=$ mean particle-mass density

$R_{j}=$ jet radius

$r=$ radial distance

$s=$ a global time variable

$\boldsymbol{U}=$ particle velocity

$\boldsymbol{V}=$ sample space variable for $\boldsymbol{U}$

$\boldsymbol{W}=$ isotropic Wiener process

$\boldsymbol{X}=$ particle position

$x=$ axial distance

$\boldsymbol{x}=$ sample space variable for $\boldsymbol{X}$

$\delta=$ Dirac delta function

$\mu=$ numerical weight

$\xi_{x}=$ grid-stretching factor in $x$ direction

$\xi_{y}=$ grid-stretching factor in $y$ direction

$\Omega=$ conditional mean turbulent frequency

\langle\rangle$=$ mean field

$=$ rms fluctuating quantity

$=$ mass-weighted field

\section{Introduction}

$\mathbf{T}$ IME-MARCHING methods have become a popular technique for the solution of steady problems in computational fluid dynamics. The main advantage of the time-marching method is that it offers the freedom of altering the time-dependent nature of

Received 10 August 2001; revision received 11 February 2002; accepted for publication 11 February 2002. Copyright (C) 2002 by the American Institute of Aeronautics and Astronautics, Inc. All rights reserved. Copies of this paper may be made for personal or internal use, on condition that the copier pay the $\$ 10.00$ per-copy fee to the Copyright Clearance Center, Inc., 222 Rosewood Drive, Danvers, MA 01923; include the code 0001-1452/02 $\$ 10.00$ in correspondence with the CCC.

*Research Associate, Sibley School of Mechanical and Aerospace Engineering; currently Assistant Professor, Department of Mechanical Engineering, Koc University, Sariyer, Istanbul 80910, Turkey; mmuradoglu@ku.edu.tr.

${ }^{\dagger}$ Professor, Sibley School of Mechanical and Aerospace Engineering. Associate Fellow AIAA. the solution as long as the problem remains well posed and the steady solution is not affected. This freedom forms the basis for many convergence acceleration techniques widely used in Eulerian time-marching algorithms such as the preconditioning ${ }^{1}$ and local time-stepping ${ }^{2}$ methods. Of these convergence acceleration methods, local time stepping has long been used as a standard technique to improve the numerical efficiency of conventional Eulerian timemarching schemes due to its conceptual simplicity and ease of implementation. The basic idea of local time stepping is to advance the solution in pseudotime using a spatially varying local time step determined according to the stability considerations at each grid point. It is inherently straightforward and easy to implement the method in Eulerian time-marching algorithms because the time derivative terms in the Eulerian formulation of the flow equations appear as partial derivatives that vanish in a steady state. However, this is not the case in a Lagrangian method. The difference is that the time derivative terms in the Lagrangian formulation of the flow equations appear as full derivatives representing both the time evolution and convection and (in general) do not vanish in the steady state. Therefore, application of local time stepping in a Lagrangian timemarching scheme is more difficult than in the Eulerian counterpart and requires the development of a new algorithm.

The main purpose of the present study is to develop and demonstrate the local time-stepping algorithm to accelerate the convergence rate of the Lagrangian particle method used to solve the probability density function(PDF) equations of turbulent reacting flows. In the PDF methods considered here, turbulenceclosure is achieved through construction of stochastic differential equations, ${ }^{3}$ and the resulting modeled PDF transport equation is solved by either a selfcontained Lagrangian particle-based Monte Carlo method (such as that implemented in the PDF2DV code ${ }^{\ddagger}$ or by a hybrid Eulerian finite volume $(\mathrm{FV}) /$ Lagrangian particle-based Monte Carlo method ( such as that implemented in the HYB2D code ${ }^{4,5}$ ). The consistent hybrid FV/particle method has recently been developed and shown to be superior to the self-contained particle/mesh method in terms of numerical efficiency. ${ }^{4,5}$ Although the present local time-stepping method is general and applicable to both PDF solution algorithms mentioned, here the method is described in the context of the consistent hybrid solution algorithm.

The consistent hybrid algorithm combines the best features of an Eulerian FV method and a Lagrangian particle-based Monte Carlo method to solve efficiently the modeled PDF transport equations of

¥Pope, S. B., "PDF2DV: A FORTRAN Code for Solving Modeled JointPDF Equations in Two-Dimensions," Sibley School of Mechanical and Aerospace Engineering, Cornell University, Ithaca, NY, 1994 (unpublished). 
turbulent reacting flows. A FV scheme is used to solve the mean mass, mean momentum, and mean energy conservation equations coupled with the mean equation of state; and a particle-based Monte Carlo method is used to solve the modeled transport equation of the joint PDF for fluctuating velocity, turbulent frequency, and compositions. The details of the consistent hybrid method may be found in Refs. 4 and 5.

The present time-stepping method is developed to accelerate the convergencerate of the Lagrangian particle method employed in the consistenthybrid algorithm, that is, to reach the steady-statein fewer time steps than are required in the conventionaluniform (or constant) time-stepping method. The main idea is to alter the time-dependent nature of the modeled PDF transport equation such that it allows a variable time step to be used in the particle method while keeping the steady-state solution unaffected. The method is very simple to implement and requires negligible additional computational work. A similar local time-stepping method has been recently presented by Mobus et al. ${ }^{6}$ for convergence acceleration of Lagrangian PDF methods but without giving any mathematical basis.

The local time-steppingmethod is incorporatedinto the consistent hybrid algorithm implemented in the code HYB2D, ${ }^{5}$ and its performance is evaluated in terms of numerical accuracy and efficiency using the standard test case of a nonreacting bluff-body flow. This flow has been studied previously experimentallyby Dally et al. ${ }^{7}$ and recently by Kalt and Masri ${ }^{\S}$ and numerically using the same PDF method by Jenny et al. ${ }^{8}$

In the next section, the PDF formulation and the consistent hybrid algorithm are briefly described, and then the local time-stepping algorithm is presented. In Sec. III, the test problem is described, and the results are presented to show the validity and benefits of the local time-stepping algorithm. Finally, conclusions are drawn in Sec. IV.

\section{Development of the Local Time-Stepping Algorithm \\ A. PDF Formulation}

The PDF formulation is based on the modeled transport equation for the joint PDF of velocity, turbulence frequency and compositions. Taking a Lagrangian viewpoint, the flow is represented by a large number of stochastic particles, and stochastic differential equations are constructed to model the evolution of the particle properties. The evolution of the particle velocity and turbulent frequency are represented by the simplified Langevin model (see Ref. 3) and the modified Jayesh-Pope model (see Van Slooten et al. ${ }^{10}$ ), respectively. All of the model constants are set to the same values as those used by Jenny et al. ${ }^{8}$ The evolution of particle composition due to molecular mixing is modeled by a mixing model, but this is not an issue here because only a constant density flow is considered in the present study. A complete description of the PDF formulation may be found in Refs. 9 and 11.

The modeled transport equation of the joint PDF is solved by the consistent hybrid method implemented in the code HYB2D. ${ }^{4,5}$ The hybrid method has been developed to overcome the deficiencies of the much-used stand-alone particle/mesh method, that is, to reduce the deterministic bias error and to avoid the complicated pressurecorrection algorithm. In the hybrid approach, a FV method is used to solve the conservation equations for mean mass, mean momentum, and mean energy coupled with the mean equation of state; a particle-based Monte Carlo method is used to solve the PDF transport equation for the fluctuating velocity, turbulent frequency, and compositions. The FV and particle algorithms are loosely coupled, that is, to complete one outer iteration, the FV code is run for a specified number of time steps and then the particle code is run for a specified number of time steps. Unlike some earlier hybrid methods, the present method is completely consistent at the level of governing equations. Furthermore, efficient correction algorithms have recently been developed to enforce full consistency at the numerical solution level. ${ }^{5}$ The consistenthybrid method has been shown to be more efficient computationally than the stand-alone particle/mesh

\footnotetext{
${ }^{\S}$ Combustion database, University of Sydney, Sydney, New South Wales, Australia, and Combustion Research Facility, Sandia National Laboratories, available online at http://www.aeromech.eng.usyd.edu.au/research/energy.
}

method by a factor of an order of magnitude or more. The details of the hybrid method may be found in Refs. 4 and 5.

\section{B. Outline of the Development}

The present hybrid method implemented in the code HYB2D is designed to treat only statistically steady turbulent flows, and the numerical solution procedure is based on a pseudo-time-marching scheme. Therefore, we are free to alter the transient time-dependent nature of the solution procedure to accelerate the global convergence of the algorithm. A novel local time-stepping algorithm is described for statistically steady solutions of PDF equations in the Lagrangian formulation. The development follows that of Pope. ${ }^{\text {II }}$

In the next subsection, the standard particle equations using uniform time stepping are presented and analyzed. The one-pointstatistics are characterized by the mass-density function $\mathcal{F}(\boldsymbol{V}, \boldsymbol{x}, t)$, the conservation equation for which is derived from the particle equations. Then the particle equations appropriatefor local time stepping are introduced and analyzed. In this case, the mass-density function is denoted by $\hat{\mathcal{F}}(\boldsymbol{V}, \boldsymbol{x}, s)$, where $s$ is a global time variable. The basis of the development is to construct the local time-steppingparticle equations so that the conservation equations for $\mathcal{F}$ and $\hat{\mathcal{F}}$ become identical in the steady state $(\partial \mathcal{F} / \partial t=0$ and $\partial \hat{\mathcal{F}} / \partial s=0)$.

\section{Analysis of Uniform Time Stepping}

We consider the standard uniform (or constant) time-stepping approach. The general particle has mass $m$, numerical weight $\mu=1$, position $\boldsymbol{X}(t)$, and velocity $\boldsymbol{U}(t)$. The model equations are

$$
\begin{gathered}
\frac{\mathrm{d} \mu}{\mathrm{d} t}=0 \\
\frac{\mathrm{d} m}{\mathrm{~d} t}=0 \\
\frac{\mathrm{d} \boldsymbol{X}}{\mathrm{d} t}=\boldsymbol{U} \\
\mathrm{d} \boldsymbol{U}=\boldsymbol{A}(\boldsymbol{U}, \boldsymbol{X}, t) \mathrm{d} t+[2 B(\boldsymbol{U}, \boldsymbol{X}, t)]^{\frac{1}{2}} \mathrm{~d} \boldsymbol{W}
\end{gathered}
$$

The first three equations express that the weight and mass of a particle are conserved and that the particle moves with its own velocity. (Note that, in practical implementations, the particles are split and clustered for the purpose of particle number control, but this has no effects on mass conservation in detail at the particle level nor on the analysis presented subsequently.) Equation (4) is a stochastic differential equation with drift term coefficient $\boldsymbol{A}$ and isotropic diffusion term coefficient $B$ : This is of the same form as the generalized Langevin model, which is usually used in PDF methods. The final quantity in Eq. (4), dW is the infinitesimal increment of an isotropic Wiener process.

The mass-density function

$$
\mathcal{F}(\boldsymbol{V}, \boldsymbol{x}, t)=\langle\mu m \delta(\boldsymbol{U}-\boldsymbol{V}) \delta(\boldsymbol{X}-\boldsymbol{x})\rangle
$$

provides a complete one-point statistical description of the particle system. In Eq. (5), $\boldsymbol{V}$ and $\boldsymbol{x}$ are the sample space variables for the particlevelocity and position, respectively. The particle mass density is

$$
q(\boldsymbol{x}, t) \equiv \int \mathcal{F} \mathrm{d} \boldsymbol{V}=\langle\mu m \delta(\boldsymbol{X}-\boldsymbol{x})\rangle
$$

which corresponds to the mean fluid density, and then the massweighted PDF of velocity is

$$
\tilde{f}(\boldsymbol{V}, \boldsymbol{x}, t)=\frac{\mathcal{F}(\boldsymbol{V}, \boldsymbol{x}, t)}{q(\boldsymbol{x}, t)}
$$

\footnotetext{
"Pope, S. B., "Variable Time Step Particle Method for Statiscally Stationary Flows," Sibley School of Mechanical and Aerospace Engineering, Cornell University, Ithaca, NY, 1995 (unpublished note).
} 
The evolution equation for the mass density function can be derived from Eqs. (1-5) by standard techniques..$^{11}$ The result is

$$
\frac{\partial \mathcal{F}}{\partial t}=-\frac{\partial}{\partial V_{i}}\left[\mathcal{F} A_{i}\right]-\frac{\partial}{\partial x_{i}}\left[\mathcal{F} V_{i}\right]+\frac{\partial^{2}}{\partial V_{i} \partial V_{i}}[\mathcal{F} B]
$$

where $A_{i}=A_{i}(\boldsymbol{V}, \boldsymbol{x}, t)$ and $B=B(\boldsymbol{V}, \boldsymbol{x}, t)$. In a statisticallystationary state, the left-hand side, $\partial \mathcal{F} / \partial t$, is zero [even though the particle properties $\boldsymbol{X}(t)$ and $\boldsymbol{U}(t)$ continue to evolve in time].

\section{Analysis of Local Time Stepping}

To develop the local time-stepping method, we replace $t$ by a global time variable $s$, so that the particle properties are $m, \mu(s)$, $\boldsymbol{X}(s)$, and $\boldsymbol{U}(s)$. A local time increment is then given by

$$
\mathrm{d} t=\gamma(\boldsymbol{x}, s) \mathrm{d} s
$$

where $\gamma(\boldsymbol{x}, s)$ is a specified, strictly positive, nondimensional field. The particle evolution equations are then specified to be

$$
\begin{gathered}
\frac{\mathrm{d} m}{\mathrm{~d} s}=0 \\
\frac{\mathrm{d} \mu}{\mathrm{d} s}=\gamma \beta \mu \\
\frac{\mathrm{d} \boldsymbol{X}}{\mathrm{d} s}=\gamma \boldsymbol{U} \\
\mathrm{d} \boldsymbol{U}=\gamma \boldsymbol{A} \mathrm{d} s+\sqrt{2 \gamma B} \mathrm{~d} \boldsymbol{W}
\end{gathered}
$$

where the field $\beta(\boldsymbol{U}, \boldsymbol{x}, s)$ is determined subsequently. Note that for $\gamma=1$ and $\beta=0$ these equations revert to their constant time-step counterparts, Eqs. (1-4). For this system, the mass-density function is defined by

$$
\hat{\mathcal{F}}(\boldsymbol{V}, \boldsymbol{x}, s)=\langle\mu m \delta(\boldsymbol{U}-\boldsymbol{V}) \delta(\boldsymbol{X}-\boldsymbol{x})\rangle
$$

When standard techniques ${ }^{11}$ are used, it follows from Eqs. (10-14) that the mass-density function evolves by

$$
\frac{\partial \hat{\mathcal{F}}}{\partial s}=\hat{\mathcal{F}} \gamma \beta-\frac{\partial}{\partial V_{i}}\left[\gamma A_{i} \hat{\mathcal{F}}\right]-\frac{\partial}{\partial x_{i}}\left[\hat{\mathcal{F}} V_{i} \gamma\right]+\frac{\partial^{2}}{\partial V_{i} \partial V_{i}}[\hat{\mathcal{F}} B \gamma]
$$

Dividing Eq. (15) by $\gamma$, we obtain

$$
\frac{1}{\gamma} \frac{\partial \hat{\mathcal{F}}}{\partial s}-\hat{\mathcal{F}}\left\{\beta-\frac{V_{i}}{\gamma} \frac{\partial \gamma}{\partial x_{i}}\right\}=-\frac{\partial}{\partial V_{i}}\left[\hat{\mathcal{F}} A_{i}\right]-V_{i} \frac{\partial \hat{\mathcal{F}}}{\partial x_{i}}+\frac{\partial^{2}}{\partial V_{i} \partial V_{i}}[\hat{\mathcal{F}} B]
$$

It may be observed that the right-hand side of this equation is identical to that of the corresponding constant time-step equation, Eq. (6). Hence, if $\beta$ can be specified so that the left-hand side of Eq. (16) vanishes in the steady state, then Eqs. (8) and (16) yield the same steady-state solutions.

The field $\beta(\boldsymbol{U}, \boldsymbol{x}, t)$ determines how the numerical weight $\mu(s)$ evolves [Eq. (11)]. We show now that the appropriate behavior is for $\mu(s)$ to remain in a fixed proportion to $\gamma[\boldsymbol{X}(s), s]$ as the particle moves. This specification implies

$$
\frac{\mathrm{d}}{\mathrm{d} s}\left\{\frac{\mu(s)}{\gamma[\boldsymbol{X}(s), s]}\right\}=0
$$

from which we obtain

$$
\frac{1}{\gamma} \frac{\mathrm{d} \mu}{\mathrm{d} s}=\frac{\mu}{\gamma^{2}}\left(\frac{\partial \gamma}{\partial s}+\frac{\mathrm{d} \boldsymbol{X}}{\mathrm{d} s} \cdot \nabla \gamma\right)
$$

or [making use of Eqs. (11) and (12)]

$$
\beta \equiv \frac{1}{\gamma \mu} \frac{\mathrm{d} \mu}{\mathrm{d} s}=\frac{1}{\gamma^{2}}\left(\frac{\partial \gamma}{\partial s}+\gamma V_{i} \frac{\partial \gamma}{\partial x_{i}}\right)
$$

Substituting this expression for $\beta$ into Eq. (16), we obtain

$$
\frac{\partial}{\partial s}\left(\frac{\hat{\mathcal{F}}}{\gamma}\right)=-\frac{\partial}{\partial V_{i}}\left[\hat{\mathcal{F}} A_{i}\right]-\frac{\partial}{\partial x_{i}}\left[\hat{\mathcal{F}} V_{i}\right]+\frac{\partial^{2}}{\partial V_{i} \partial V_{i}}[\hat{\mathcal{F}} B]
$$

Compare Eq. (8). In the steady state, the left-hand sides of Eqs. (8) and (20) vanish, whereas their right-hand sides are identical. Thus, the steady-state solution to the local time-step equations are statistically identical to those of the standard uniform time-step system.

From the preceding development, we can draw the following conclusions:

First, in the modified particle equations [Eqs. (10-13)], the particle properties are indexed by the global time variable $s$. The increments in the properties $\boldsymbol{X}$ and $\boldsymbol{U}$ in the infinitesimal time ds corresponds to those in the uniform time-stepping equations [Eqs. (1-4)] over a time interval $\mathrm{d} t=\gamma \mathrm{d} s$. Thus, if $\Delta s$ is the global time step, the local time step is $\Delta t=\gamma(\boldsymbol{x}, s) \Delta s$. For example, a finite difference approximation to Eq. (13) is

$$
\Delta \boldsymbol{U} \equiv \boldsymbol{U}(s+\Delta s)-\boldsymbol{U}(s)=\boldsymbol{A}[\gamma \Delta s]+\sqrt{2 B[\gamma \Delta s]} \boldsymbol{\xi}
$$

where $\boldsymbol{\xi}$ is a standardized Gaussian random vector.

Second, for each particle, Eq. (17) can be integrated from $s_{1}$ to $s_{2}$ to yield

$$
\mu\left(s_{2}\right)=\mu\left(s_{1}\right) \frac{\gamma\left[\boldsymbol{X}\left(s_{2}\right), s_{2}\right]}{\gamma\left[\boldsymbol{X}\left(s_{1}\right), s_{1}\right]}
$$

showing that the change in particle weight is simply determined by the values of $\gamma$ at the start and end of the particle trajectory. This is exact even if $\gamma$ is piecewise constant, for example, constant within each spatial cell.

Third, the uniform time-steppingmethod is conservativein detail, in that the effective particle mass $\mu m$ is conserved. Consequently, conservation of mass, momentum, species, etc., can be enforced in detail at the particle level, not just in the mean. In the local timestepping method, this detailed conservation property is lost because the effective particle mass $\mu(s) m$ is not conserved. As the results in the next section demonstrate, this lack of detailed conservation does not appear to affect adversely the accuracy of the calculations.

Fourth, the particle equations considered [Eqs. (1-4)] are not the most general of interest. In particular, if molecular viscosity is considered, the equation for $\boldsymbol{X}$ becomes a stochastic differentialequation with a diffusion term related to the molecular viscosity. ${ }^{12}$ Similarly, in the composition PDF method, the SDE for $\boldsymbol{X}$ contains a diffusion term related to the turbulent diffusivity. ${ }^{3,11}$ In these cases, the analysis is considerably more involved, but the results are unchanged.

Fifth, during the transient phase of the computations, the local time step can vary with time, that is, $\gamma(\boldsymbol{x}, s)$ can depend on $s$. However, after this transient, to achieve the correct steady state, $\gamma(\boldsymbol{x}, s)$ must be fixed (independentof $s$ ), so that the left-hand side of Eq. (20) implies $\partial \hat{\mathcal{F}} / \partial s=0$.

\section{E. Specification of the Local Time Step}

Given the global time step $\Delta s$, the local time step is given by $\Delta t(\boldsymbol{x}, s)=\gamma(\boldsymbol{x}, s) \Delta s$. In practice, this relation is inverted to determine $\gamma(\boldsymbol{x}, s)$, that is, a suitable local time step $\Delta t(\boldsymbol{x}, s)$ is determined, $\Delta s$ is specified (essentially arbitrarily), and then $\gamma$ is specified as $\Delta t / \Delta s$.

The local time-stepping algorithm is incorporated into the consistent hybrid method. In the implementation, the computational domain is divided into a tensor-product rectangular mesh, and the local time step is calculated, for instance, at the $(i, j)$ grid point, as follows. The local upper bounds $\Delta t_{x_{i, j}}, \Delta t_{y_{i, j}}$, and $\Delta t_{\Omega_{i, j}}$ are defined implicitly by

$$
\begin{aligned}
C_{u} & =\frac{|U|_{i, j} \Delta t_{x_{i, j}}}{\Delta x_{i, j}} \\
C_{u} & =\frac{|V|_{i, j} \Delta t_{y_{i, j}}}{\Delta y_{i, j}}
\end{aligned}
$$




$$
C_{\Omega}=\Omega_{i, j} \Delta t_{\Omega_{i, j}}
$$

where the Courant number is specified as $C_{u}=0.4$ and the turbulentfrequency parameterby $C_{\Omega}=0.2$. Note that, unlike many numerical methods, the time step in the present method is mainly restricted by the accuracy considerations rather than the stability constraints and that the values of $C_{u}$ and $C_{\Omega}$ are essentially chosen based on the work by $\mathrm{Xu}$ and Pope. ${ }^{13}$ The conditional mean turbulent frequency $\Omega_{i, j}$ is evaluated at the $(i, j)$ grid point, and the velocity scales $|U|$ and $|V|$ are defined as

$$
\begin{aligned}
& |U|=|\langle U\rangle|+2 u^{\prime} \\
& |V|=|\langle V\rangle|+2 v^{\prime}
\end{aligned}
$$

where $\langle U\rangle,\langle V\rangle, u^{\prime}$, and $v^{\prime}$ are the axial mean velocity, the radial mean velocity, the rms axial fluctuating velocity, and the rms radial fluctuating velocity, respectively. The grid spacings $\Delta x_{i, j}$ and $\Delta y_{i, j}$ are evaluated as

$$
\begin{aligned}
\Delta x_{i, j} & =\frac{1}{2}\left(x_{i+1, j}-x_{i-1, j}\right) \\
\Delta y_{i, j} & =\frac{1}{2}\left(y_{i, j+1}-y_{i, j-1}\right)
\end{aligned}
$$

The local time step is then specified by

$$
\Delta t_{i, j}=\min \left[\Delta t_{\lim }, \Delta t_{x_{i, j}}, \Delta t_{y_{i, j}}, \Delta t_{\Omega_{i, j}}\right]
$$

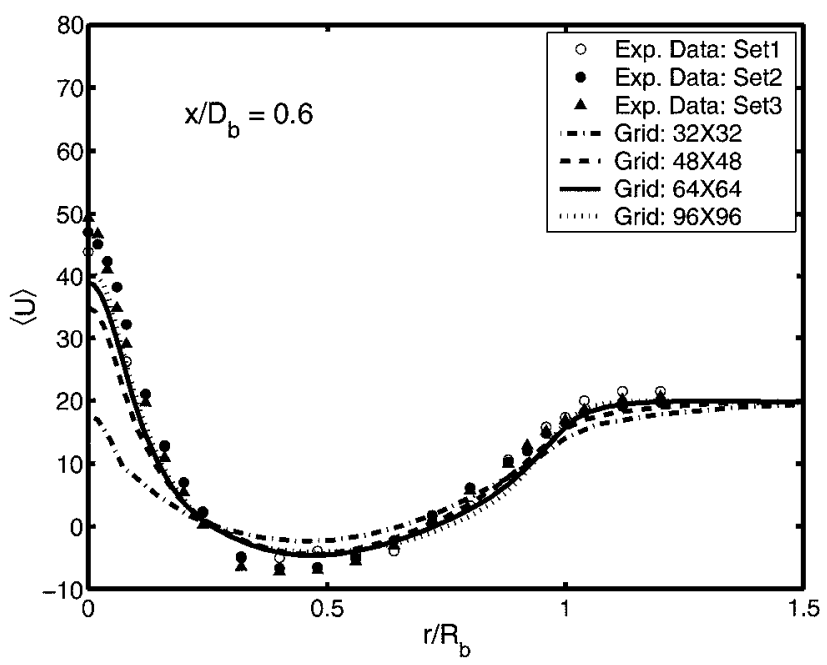

a)

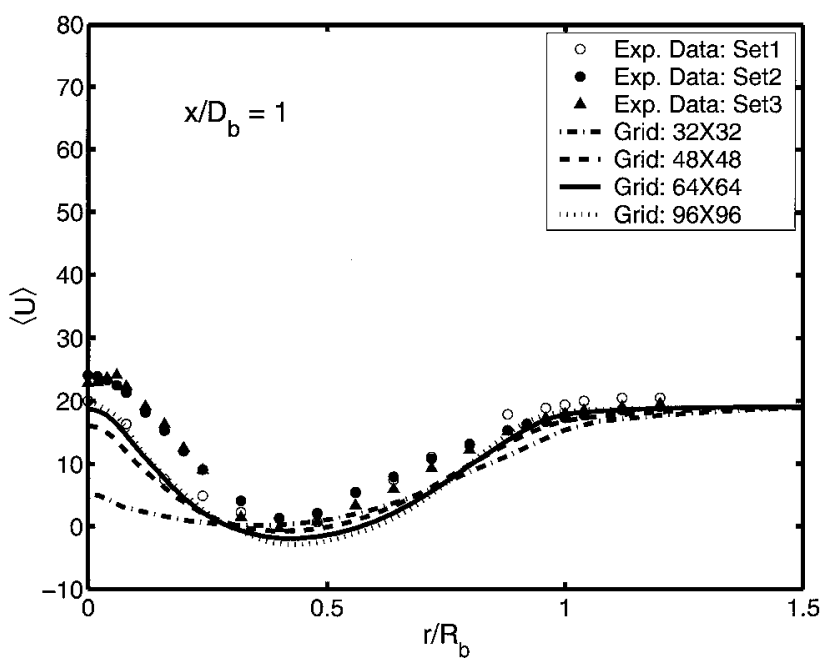

b)

Fig. 1 Profiles of mean axial velocity $(\langle U\rangle)$ computed on the most stretched grid $\left(\xi_{x}=160\right)$ at axial locations a) $0.6 D_{b}$ and b) $D_{b}$.
The parameter $\Delta t_{\text {lim }}$ sets an upper limit to the local time steps, and it is typically taken as $\Delta t_{\mathrm{lim}}=\kappa_{t} \Delta t_{\min }$, where $\Delta t_{\min }$ is the minimum value of $\Delta t_{x_{i, j}}, \Delta t_{y_{i, j}}$, and $\Delta t_{\Omega_{i, j}}$ over the entire computational domain and the parameter is taken as $\kappa_{t}=20$.

After calculating the time steps at all grid points as described, the time step is represented as a linear spline and interpolated onto the particles. The local time step is stored as a particle property and the numerical weight is updated as

$$
\mu^{n+1}=\mu^{n}\left(\Delta t^{n+1} / \Delta t^{n}\right)
$$

where superscript $n$ denotes the values evaluated at the $n$th time step. This treatment is consistent with Eq. (22) because $\mu / \gamma=\mu \Delta s / \Delta t$ is conserved following the particle.

\section{Results and Discussion}

The performance of the local time stepping method is evaluated in terms of accuracy and efficiency. The test problem chosen is the statistically axisymmetric nonreacting bluff-body flow that has been extensively studied before experimentally by Dally et al. ${ }^{7}$ and Kalt and Masri ${ }^{\S}$ and numerically using the same PDF method as employed here by Jenny et al. ${ }^{8}$ Besides its practical interest, the bluff-body flow is a challenging test case for turbulence models and numerical algorithms because it involves a strong recirculation zone with two vortex cores behind the bluff body. Both the central
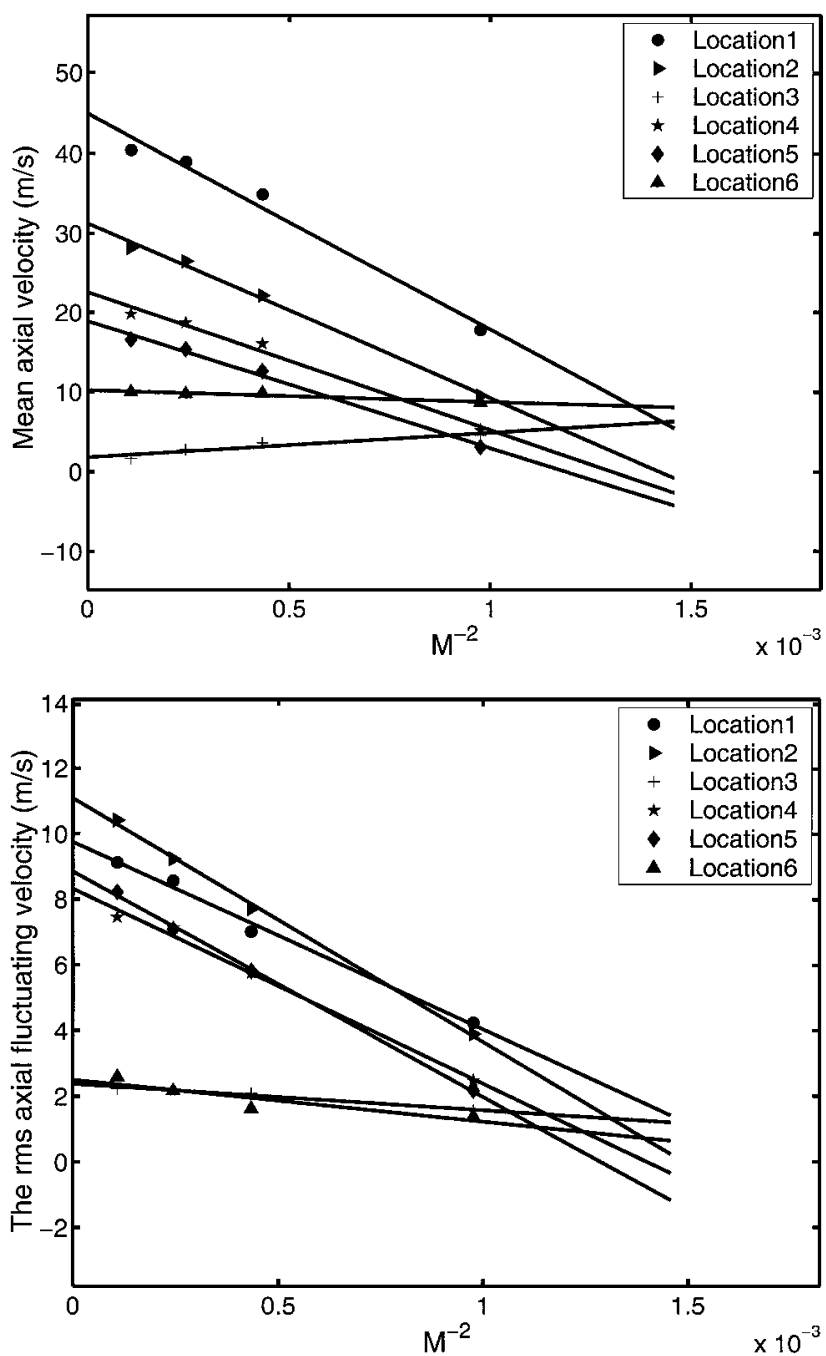

Fig. 2 Mean axial velocity and rms axial fluctuating velocity against $M^{-2}$ at the selected locations (Table 1) showing the expected secondorder spatial accuracy of the method: —_, linear least-square fits to numerical data. 
jet and coflow consist of cold air with bulk velocities 61 and $20 \mathrm{~m} / \mathrm{s}$, respectively. The jet diameter $2 R_{j}$ is $3.6 \mathrm{~mm}$, and the bluff body diameter $D_{b}$ is $50 \mathrm{~mm}$. The Reynolds number based on the jet bulk velocity and jet diameter is about $R e_{j}=1.44 \times 10^{4}$. The complete description of the bluff-body flow is given by Dally et al. ${ }^{7}$ The experimental data for the nonreacting bluff-body flow were first provided by Dally et al., ${ }^{7}$ and this set of data is labeled here as set 1 . The same experiment has been recently repeated twice by Kalt and Masri, ${ }^{\S}$ and these new sets of data are labeled as sets 2 and 3. It is emphasized that the same experimental set up was used for sets 2 and 3. All of the experimental data and a detailed description of the bluff-body flow are available on the Web. ${ }^{\S}$

For the numerical simulations, a cylindrical coordinate system is adopted with the origin of the radial coordinate $r$ placed at the center of the jet and the axial coordinate $x$ aligned with the jet axis. The computational domain extends in the axial direction from $x=0$ to $6 D_{b}$ and in the radial direction from $r=0$ to $3 D_{b}$. The computational domain, the initial and boundary conditions, and all of the model constants are the same as those used by Jenny et al. ${ }^{8}$ All of the computations presented here are performed on a rectangular orthogonal nonuniform Cartesian grid with a total of $M^{2}$ grid cells, and the grid is successively stretched in the axial direction to examine the performance of the local time-stepping algorithm on highly stretched grids. Note that the grid topology employed here is also the same as that of Jenny et al. ${ }^{8}$ except that the matching point in the axial direction at $x=D_{b}$ is removed in the present computations to facilitate the grid stretching. The grid stretching is quantified by the

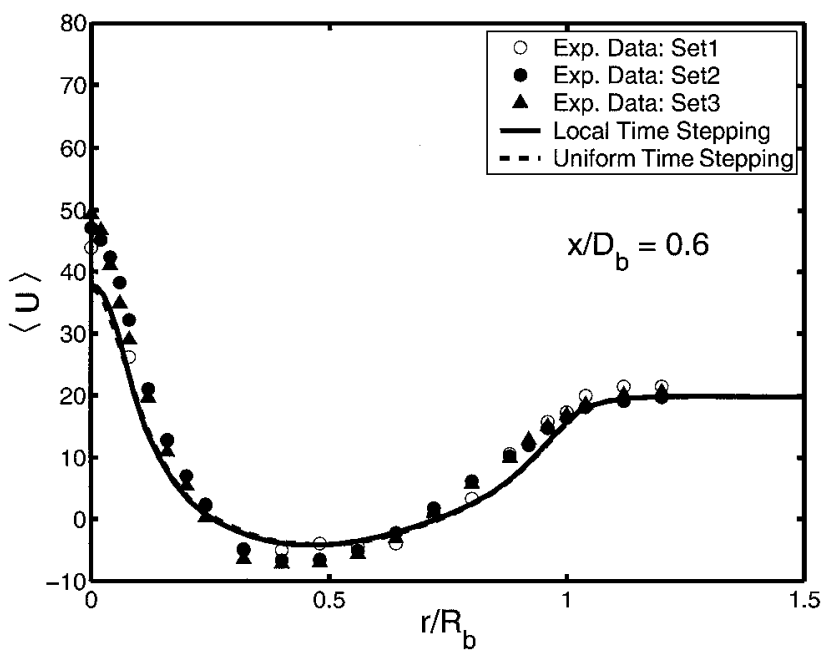

a) $x / D_{b}=0.6$

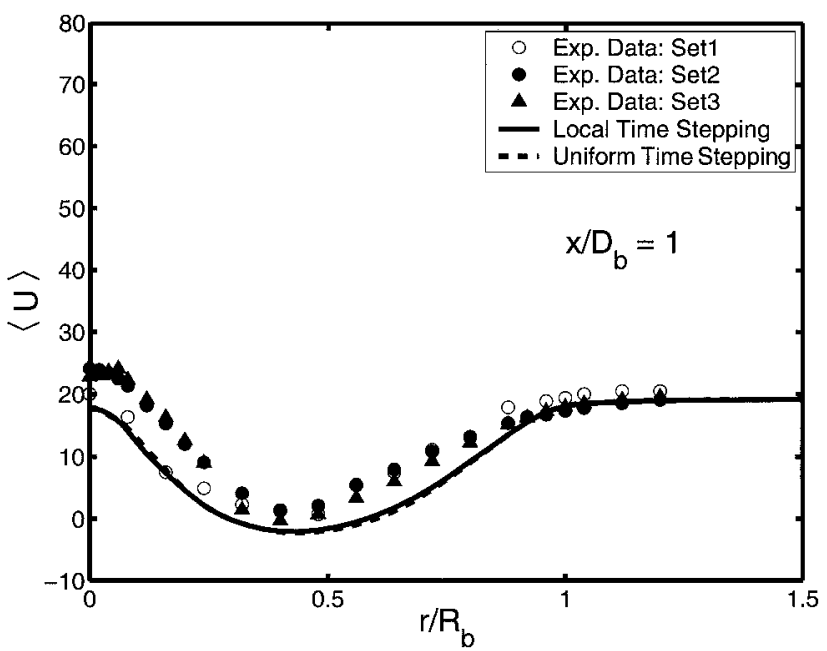

b) $x / D_{b}=1.0$

Fig. 3 Mean axial velocity profiles computed on the moderately stretched grid $\left(\xi_{x}=10\right)$. stretching rate defined as the ratio of the maximum and minimum cell sizes in each coordinate direction. For example, the stretching rate in the axial direction is defined as $\xi_{x}=\Delta x_{\max } / \Delta x_{\min }$, where $\Delta x_{\max }$ and $\Delta x_{\min }$ are the maximum and minimum cell sizes in the axial direction, respectively.

All of the mean fields are time averaged over a fixed timescale of 20 particle time steps throughout the simulations. The statistical error is reduced by using a large number of particles per cell, that is, about 200 particles per cell are used in all of the results presented here. Note that the usual practice in the consistent hybrid method is to use a larger time-averaging timescale, that is, typically over 500-1000 particle time steps and to use a smaller number of particles per cell, that is, typically $20-50$ particles per cell. Furthermore, the time-averaging timescale is not fixed in usual practice but rather increased gradually from a small value to the final large value as a steady state is approached. In the present calculations, the time-averaging timescale is chosen to be much smaller than the usual value and is fixed throughout the simulations to minimize the uncertaintiescaused by the ad hoc parametersused in the usual timeaveraging procedure and, thus, to better isolate the improvement in the numerical efficiency by the local time-stepping algorithm. The details of the consistent hybrid method and the time averaging procedure may be found in Refs. 4 and 5.

The grid convergence is shown in the next section. The accuracy of the local time-stepping method is examined in Sec. III.B, and then the improvement in the numerical efficiency is presented in Sec. III.C.

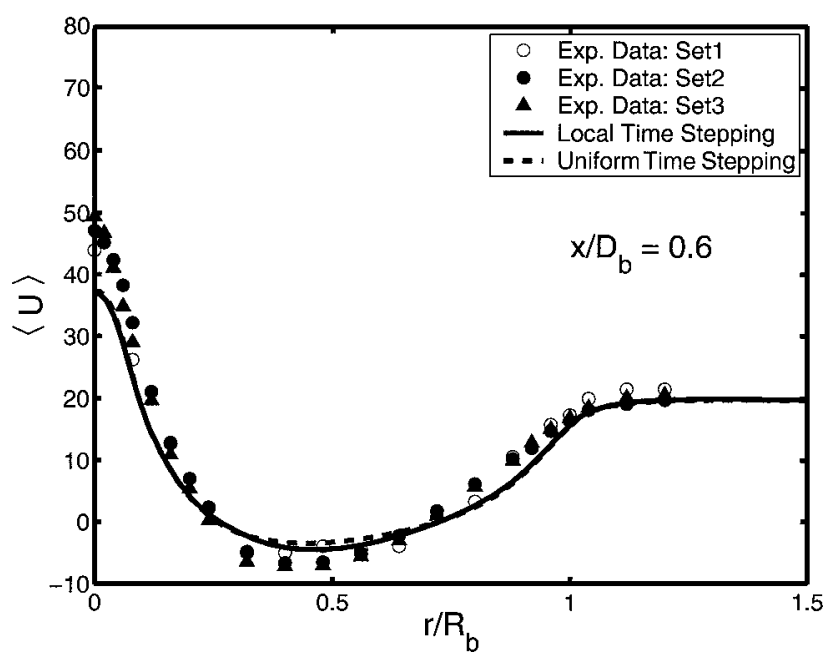

a) $x / D_{b}=0.6$

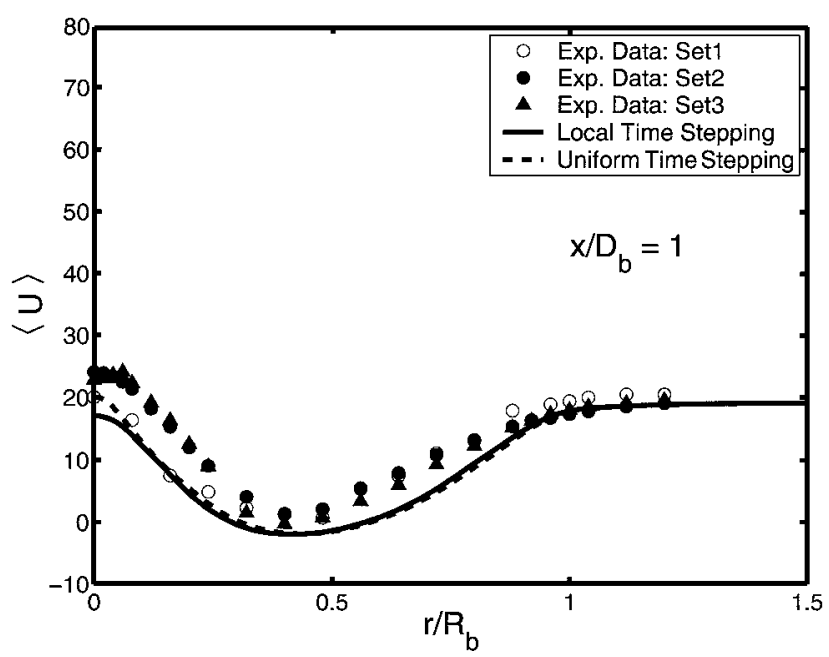

b) $x / D_{b}=1.0$

Fig. 4 Mean axial velocity profiles computed on the highly stretched grid $\left(\xi_{x}=160\right)$. 


\section{A. Grid Convergence}

The spatial discretization in the FV method together with the mean field estimation and interpolation schemes used in the particle algorithm result in spatial error in the hybrid method due to the finite size of the grid cells. The spatial error in the hybrid method has been extensively examined before, and it has been demonstrated that the method is second-order accurate in space. ${ }^{4,5}$ Therefore, the main purpose here is to show the grid convergence of the numerical results presentedin the paper rather than repeating an extensivestudy of spatial error. For this purpose, profiles of mean axial velocity are plotted in Fig. 1 at the axial locations of $x=0.6 D_{b}$ and $D_{b}$ to show overall dependence of the calculatedresults on grid refinement. Note that the calculations are performed on an extremely stretched grid for example, $\xi_{x}=160$, using the local time-stepping algorithm. As can be seen in Fig. 1, the decreasing difference between the profiles as cell is refined indicates grid convergence.

To quantify the spatial error and to verify the second-order accuracy, the mean axial velocity and rms axial fluctuating velocity are plotted against the inverse of the total number of grid cells $M^{-2}$ in Fig. 2 at the selected locations specified in Table 1. As can be seen in Fig. 2, the approximate linear relationship between the mean quantities and $M^{-2}$ confirms the expected second-order accuracy. Also note from Fig. 2 that the spatial error is less than 15 and $7 \%$ on $64 \times 64$ and $96 \times 96$ grids, respectively. Although not shown here due to space limitations, the mean axial velocity and the rms axial fluctuating velocity are found to be good representativesfor the other

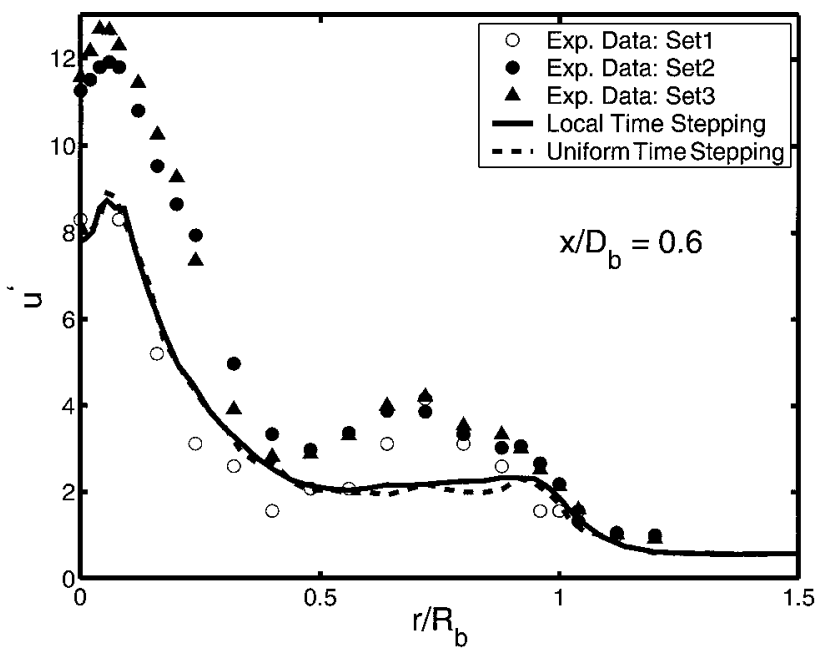

a) $x / D_{b}=0.6$

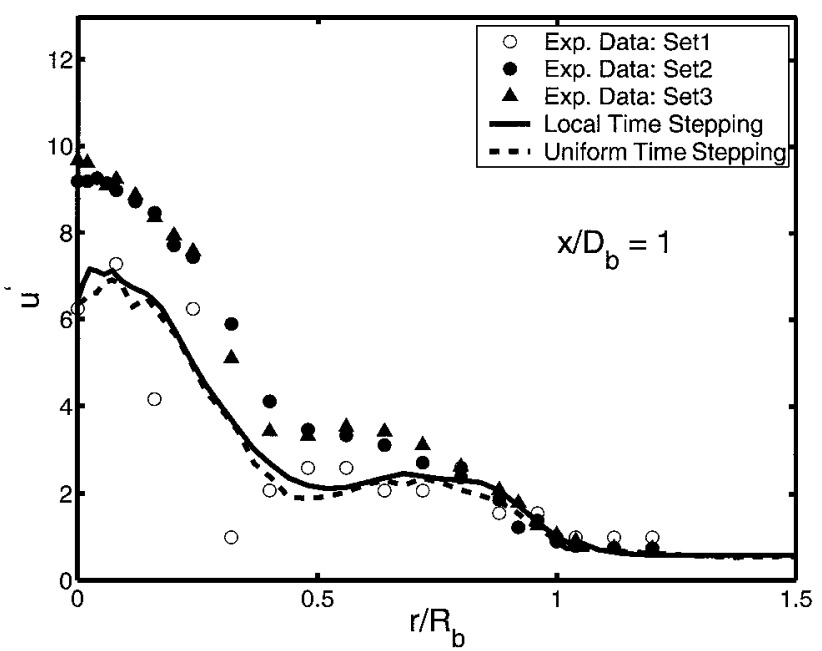

b) $x / D_{b}=1.0$

Fig. 5 RMS fluctuating axial velocity profiles computed on the moderately stretched grid $\left(\xi_{x}=10\right)$. mean quatities such as mean radial velocity, rms radial fluctuating velocity, turbulence shear stress, and mean turbulent frequency in terms of spatial accuracy and grid convergence. Because the main emphasis here is on the performance of the local time-stepping algorithm in comparison with the uniform time-stepping method, a $64 \times 64$ grid is used in all of the results presented in the rest of the paper.

\section{B. Accuracy}

As discussed in Sec. II, the local time-stepping method alters the time-dependent nature of the solutions but guarantees that the steady-state solutions remain unaffected. The accuracy of the local time-stepping method is evaluated through comparison of the

Table 1 Six selected locations in the computational domain used to quantify spatial error

\begin{tabular}{lcc}
\hline \hline Location & Axial distance $x$ & Radial distance $r$ \\
\hline 1 & $0.6 D_{b}{ }^{\mathrm{a}}$ & 0.0 \\
2 & $0.6 D_{b}$ & $R_{j}^{\mathrm{b}}$ \\
3 & $0.6 D_{b}$ & Mid bluff body $(=13.4 \mathrm{~mm})$ \\
4 & $D_{b}$ & 0.0 \\
5 & $D_{b}$ & $R_{j}$ \\
6 & $D_{b}$ & Mid bluff body $(=13.4 \mathrm{~mm})$ \\
\hline \hline
\end{tabular}

${ }^{\mathrm{a}} D_{b}=50 \mathrm{~mm}$ is bluff-body diameter.

${ }^{\mathrm{b}} R_{j}=1.8 \mathrm{~mm}$ is jet radius.

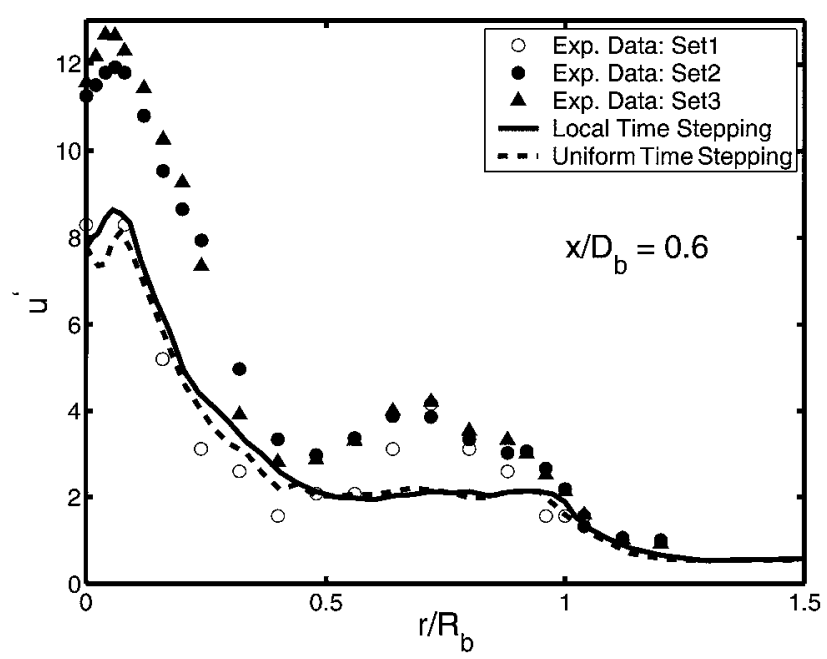

a) $x / D_{b}=0.6$

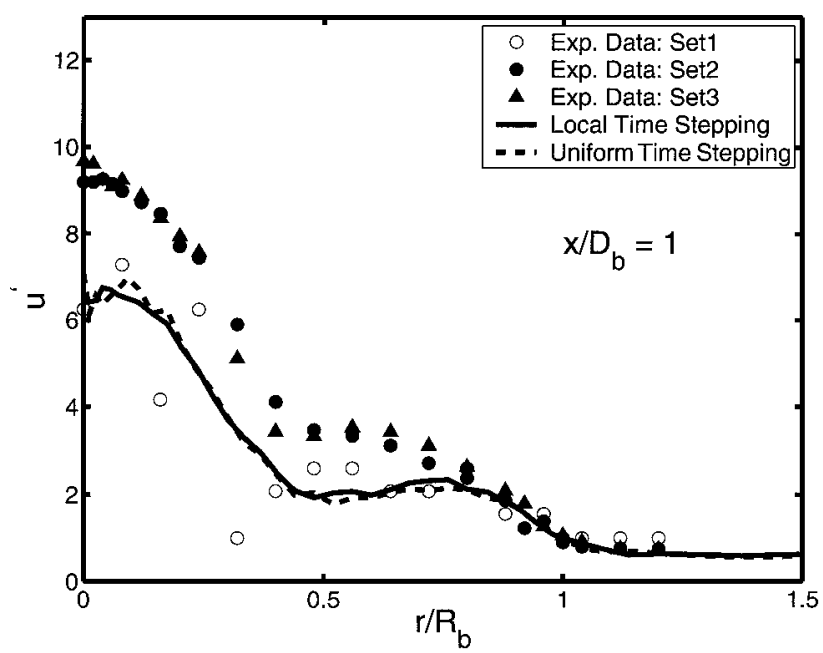

b) $x / D_{b}=1.0$

Fig. 6 RMS fluctuating axial velocity profiles computed on the highly stretched grid $\left(\xi_{x}=160\right)$. 
mean axial velocity and the rms axial fluctuating velocity profiles calculated using the local and uniform time-stepping methods. Such comparisons are made for two sets of computational grids with different stretching rates to show the effects of the grid stretching on the accuracy of the local time-stepping method. The first grid is very similar to that used by Jenny et al., ${ }^{8}$ and the stretching rates in the axial and radial directions are $\xi_{x}=10$ and $\xi_{y}=30$, respectively. The second grid is highly stretched in the axial direction with the stretching rate $\xi_{x}=160$, whereas the stretching rate is kept the same as the first grid in the radial direction. The first and the second grids are referred to from now on as the least and the most stretched grids, respectively. Although it is not emphasized here, the experimental data are also plotted and compared with the numerical results.

Figures 3 and 4 show the mean axial velocity profiles calculated using the least and the most stretched grids, respectively, at the axial locations $x=0.6 D_{b}$ (Figs. 3a and $4 \mathrm{a}$ ) and $x=D_{b}$ (Figs. 3b and 4b). In Figs. 3 and 4, the symbols denote the experimental data and the solid and dashed lines represent the numerical results obtained with the local and uniform time-stepping methods, respectively. As can be seen in Figs. 3 and 4, even on the highly stretched grid (Fig. 4), the local time-stepping results compare very well with the corresponding uniform time-stepping results, demonstrating the accuracy of the local time-stepping algorithm.

The similar plots for the rms axial fluctuating velocity profiles are presented in Figs. 5 and 6 again for the least and the most stretched grids, respectivelyat the same axial locations of $x=0.6 D_{b}$ (Figs. 5a and 6a) and $x=D_{b}$ (Figs. 5b and 6b). As for the mean axial velocity, the excellent agreement between the local and uniform timestepping results of the rms axial fluctuating velocity confirms the accuracy of the local time-stepping method.

The small discrepancy observed between the local and uniform time-stepping results of the mean axial velocity and rms axial fluctuating velocity profiles especially near the centerline in the case of the highly stretched grid are attributed to the statistical and spatial discretization errors. We note that (not shown here due to space limitations), similar good agreement between the local and uniform time-stepping methods is observed in all of the other FV and particle mean fields such as the mean radial velocity, rms radial fluctuating velocity, mean turbulent shear stress, and mean turbulent frequency in the entire computational domain.

When it is considered that the simplest velocity model is used in the present calculations, the reasonable agreement between the numerical results and the experimental data clearly demonstrates the performance of the PDF methods for this challenging test case.

\section{Convergence Acceleration}

The primary purpose of local time stepping is to improve the numerical efficiency by accelerating the global convergence rate, that is, to converge to the steady state in fewer time steps. The global convergence of PDF solution algorithms are usually measured by monitoring the time histories of particle and FV mean fields at selected observation points in the computational domain. ${ }^{4}$ Here the

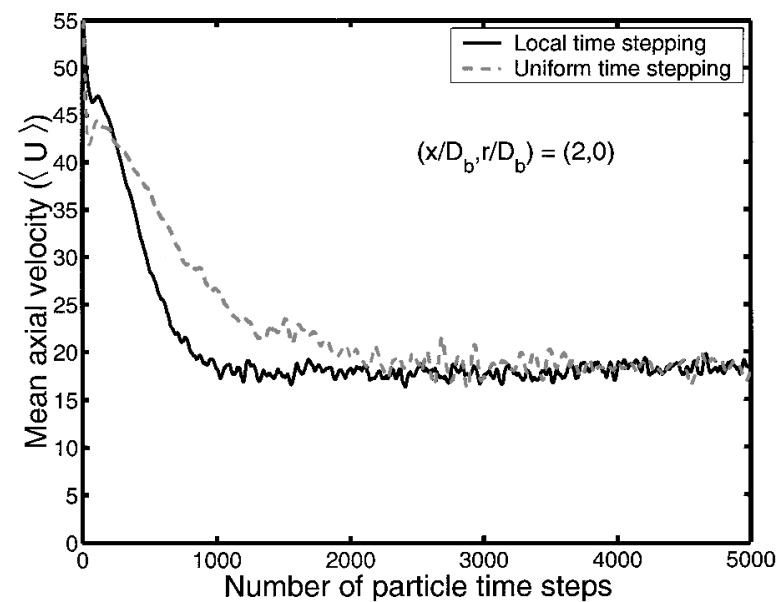

a)

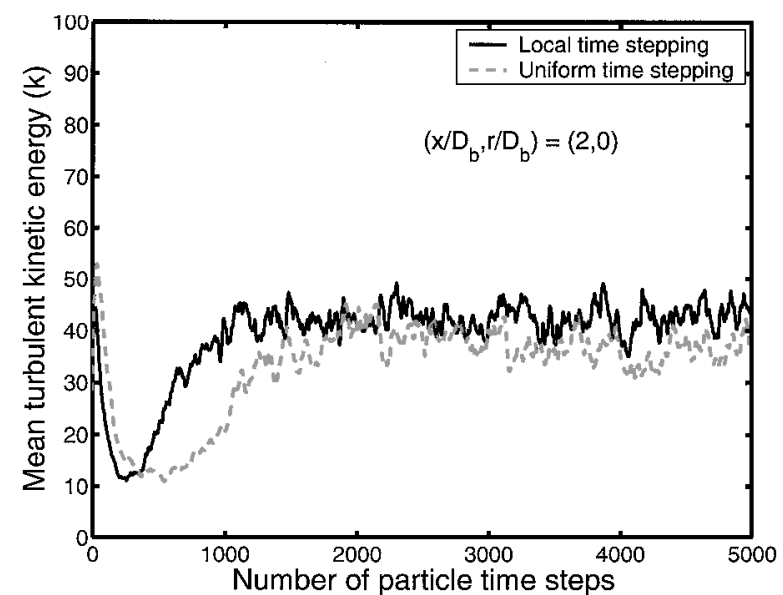

b)

Fig. 7 Moderately stretched grid $\left(\xi_{x}=10\right)$ convergence history of a) mean axial velocity at $\left(x / D_{b}, r / D_{b}\right)=(2.0,0.0)$; b $)$ mean turbulent kinetic energy at $\left(x / D_{b}, r / D_{b}\right)=(2.0,0.0)$; c) mean axial velocity at $\left(x / D_{b}, r / D_{b}\right)=(3.0,0.0) ;$ and d $)$ mean turbulent kinetic energy at $\left(x / D_{b}, r / D_{b}\right)=(3.0,0.0)$.

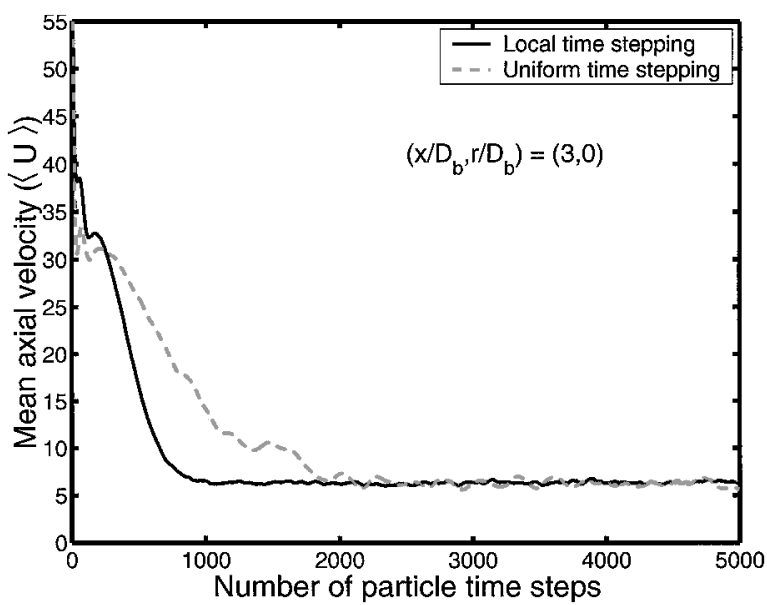

c)

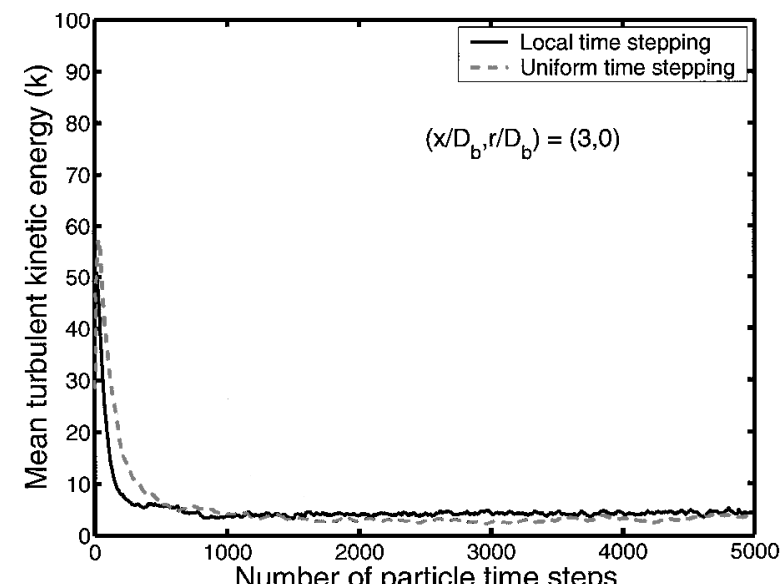

d) 
mean axial velocity and mean turbulence kinetic energy are chosen for this purpose, and their convergence histories are monitored at locations $\left(x / D_{b}, r / D_{b}\right)=(2.0,0.0)$ and $\left(x / D_{b}, r / D_{b}\right)=(3.0,0.0)$.

Figure 7 shows the convergencehistories of the mean axial velocity (Figs. 7a and 7c) and the mean turbulent kinetic energy (Figs. 7b and $7 \mathrm{~d})$ at the locations $\left(x / D_{b}, r / D_{b}\right)=(2.0,0.0)$ (Figs. 7a and 7b) and $\left(x / D_{b}, r / D_{b}\right)=(3.0,0.0)$ (Figs. $7 \mathrm{c}$ and $\left.7 \mathrm{~d}\right)$ to demonstrate the performance of the local time-stepping algorithm in accelerating the global convergence of the consistent hybrid method. The results are obtained on the least stretched grid. As can be seen in Fig. 7, even on this moderately stretched grid, the local time-stepping algorithm considerably accelerates the global convergence of the hybrid method by about a factor of 3 . For instance, whereas it takes about 3000 particle time steps with the uniform time stepping for the convergence of the mean axial velocity at $\left(x / D_{b}, r / D_{b}\right)=(2.0,0.0)$, it requires only about 1000 particle time steps with the local time stepping for this case.

The convergence acceleration by the local time stepping is expected to be more pronouncedas the grid is more and more stretched, and this is confirmed in Figs. 8 and 9. Figures 8 and 9 show the convergence histories of the mean axial velocity (Fig. 8) and mean turbulent kinetic energy (Fig. 9) calculated using the local timestepping (Figs. 8a, 8c, 9a, and 9c) and the uniform time-stepping (Figs. 8b, 8d, 9b, and 9d) methods on grids successively stretched in axial direction with the axial stretching rates $\xi_{x}=10,40$, and 160. Observe that the convergence rate is essentially independent of grid stretching when local time stepping is used, whereas, as expected, the convergence rate significantly deteriorates in the case of uniform time stepping as the grid is stretched. For example, on the most stretched grid (with the axial stretching rate $\xi_{x}=160$ ), it takes about 16,000 particle time steps to reach a steady state when the uniform time stepping is used, whereas it requires only about 1500 particle time steps in the case of local time stepping. In other words, local time stepping can accelerate the convergence rate of the consistent hybrid method by a factor of an order of magnitude depending on the grid stretching. This is clearly a significant improvement in the numerical efficiency of the consistent hybrid method.

Note that the present local time-stepping algorithmis very simple to implement, and it requires a negligible amount of additional computational work per step, that is the additional computational work per step measured in CPU time due to the local time stepping is less than $3 \%$ in the all of the results presented here. This is a negligible penalty compared to the substantial saving in the number of time steps required to reach the steady state.

Also note that the mean fields calculated using the local timestepping method are smoother than the corresponding fields calculated with the uniform time-stepping method mainly because of the accelerated convection of error waves out of the computational domain by the local time stepping. This is an important result, not only because of the smoothermean fields, but also because it significantly improves the robustness of the consistent hybrid method.

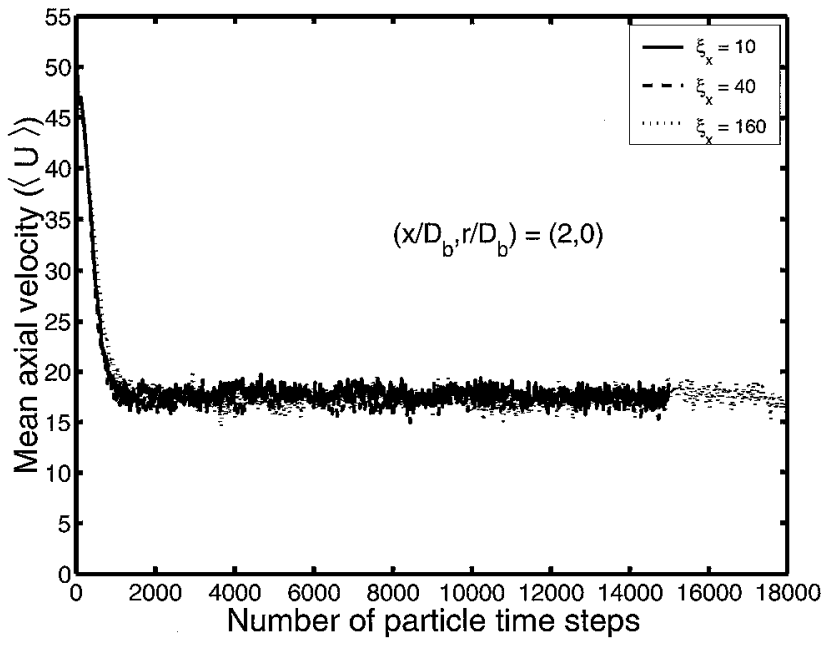

a)

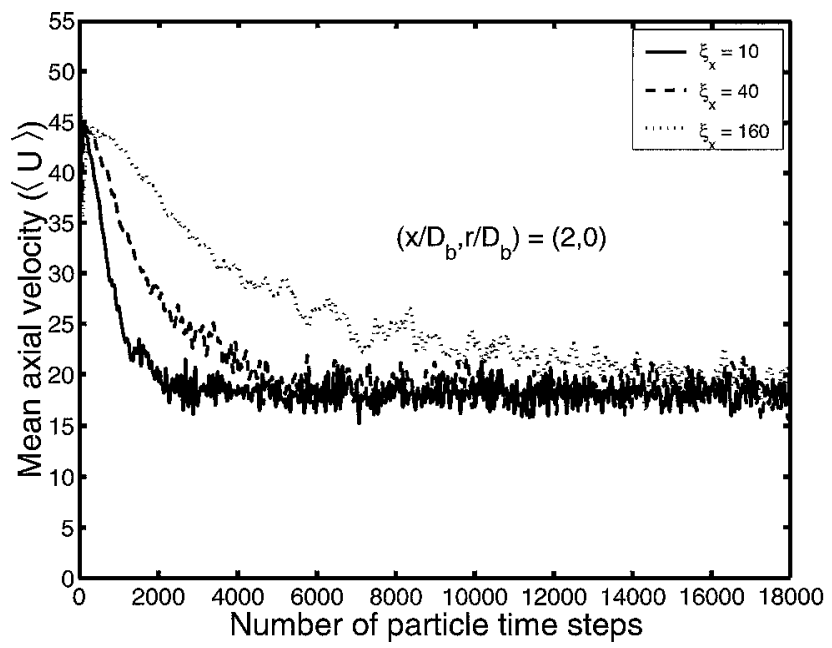

b)

Fig. 8 Convergence histories on successively stretched grids with the stretching rates from $\xi_{x}=10$ to 160 of mean axial velocity computed with a) and c) local time stepping and b) and d) uniform time stepping.

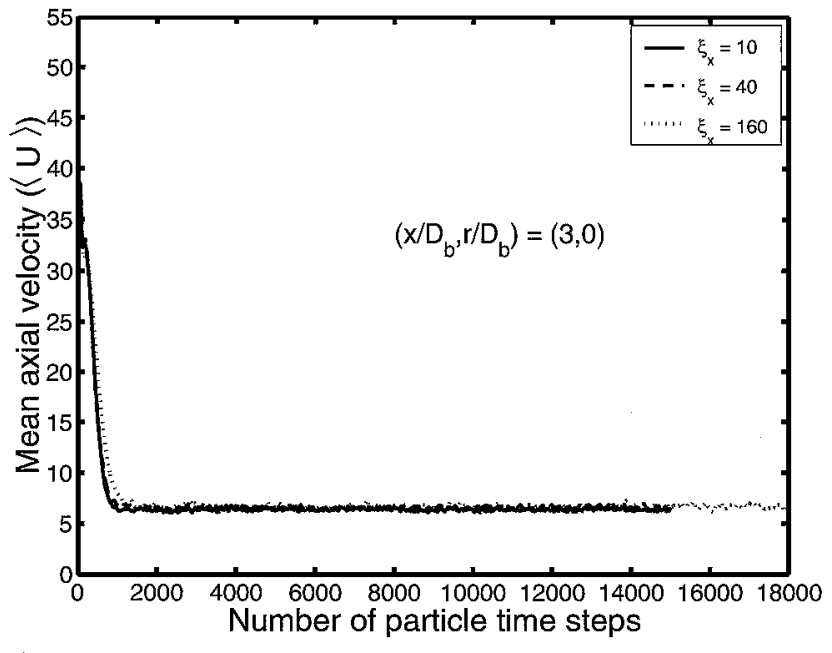

c)

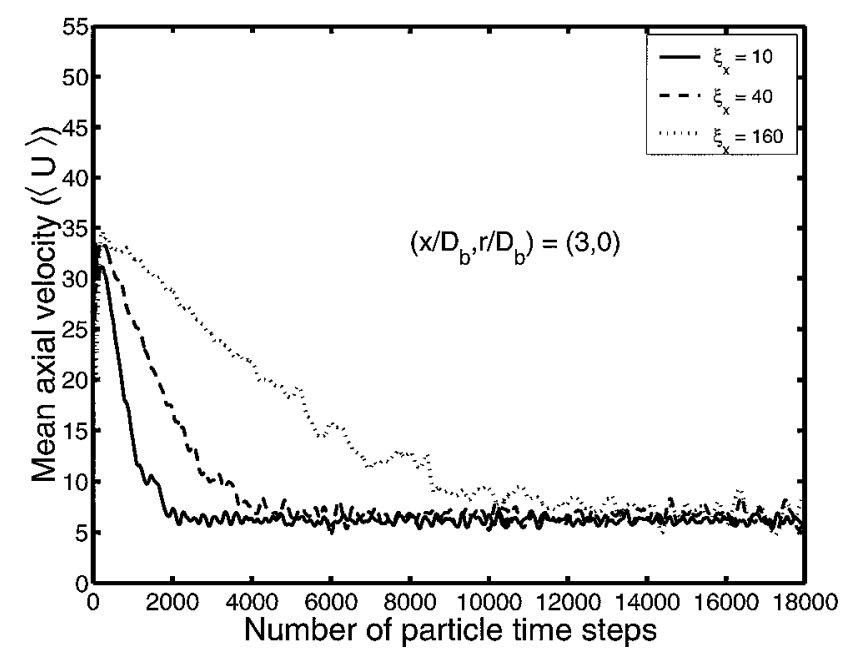

d) 


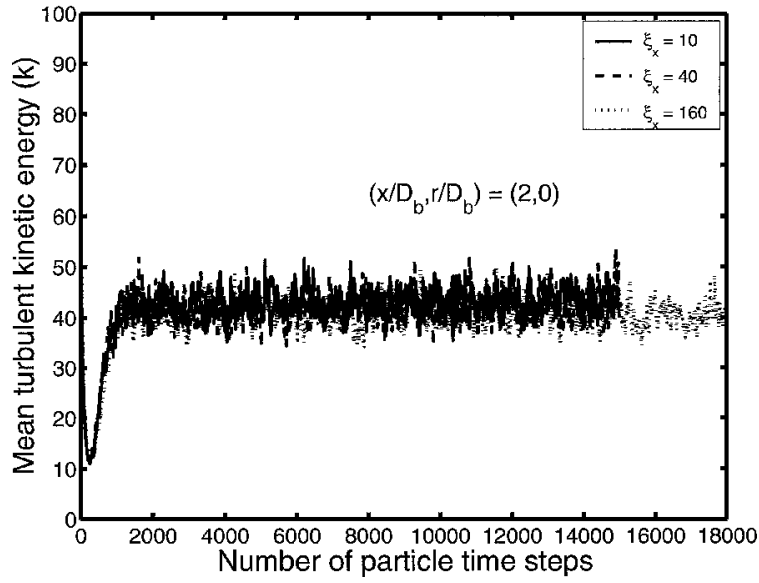

a)

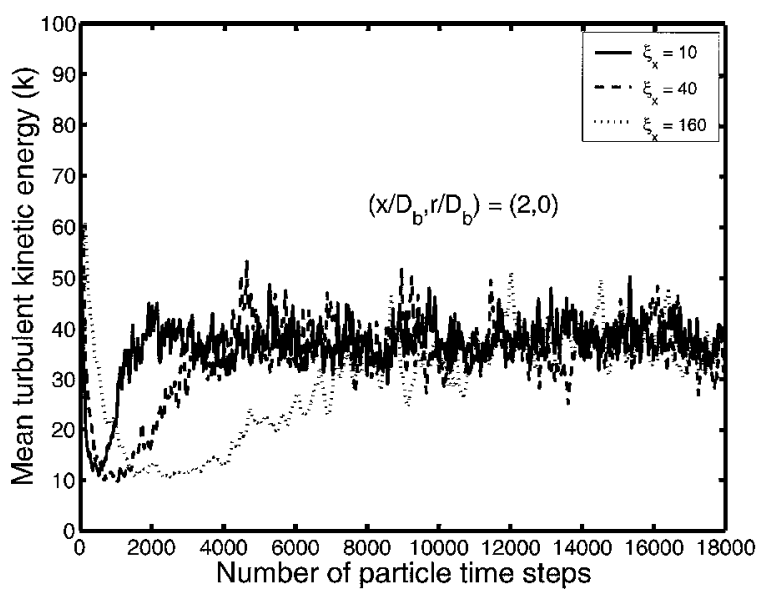

b)

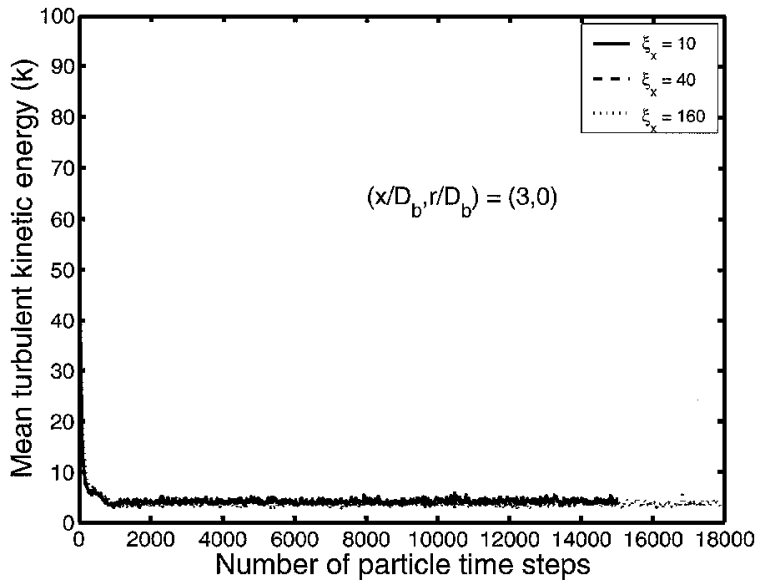

c)

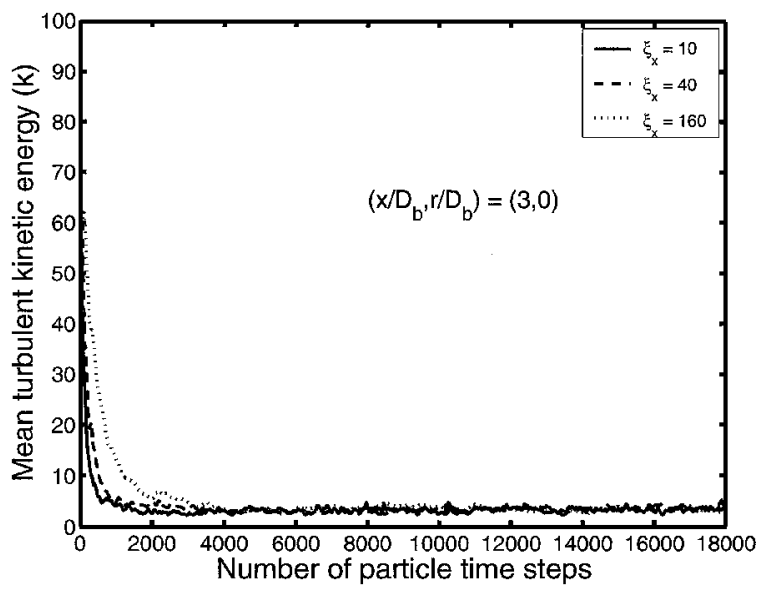

d)

Fig. 9 Convergence histories on successively stretched grids with the stretching rates from $\xi_{x}=10$ to 160 of mean turbulent kinetic energy computed with a) and c) local time stepping and b) and d) uniform time stepping.

\section{Conclusions}

A novel local time-stepping method is developed and implemented in the consistent hybrid FV/particle method to obtain statistically steady solutions of the PDF equations of turbulent reacting flows. The local time-stepping method changes the time-dependent nature of the PDF equations to allow local time steps to be used in the integration of the particle evolution equations but guarantees that the steady solutions remain unaffected. The method is tested for the challenging nonreacting bluff-body flow and is shown to accelerate significantly the global convergence of the consistent hybrid method by as much as a factor of an order of magnitude, depending on grid stretching. The accuracy of the local time-stepping method is demonstrated by comparing the mean fields calculated using the local and uniform time-stepping methods. It is found that the convergence rate of the consistenthybrid method is essentially independent of the grid stretching when local time stepping is used, whereas it significantly deteriorates in the case of uniform time stepping as the grid is stretched. It is also found that the local time stepping significantly improves the robustness of the hybrid method and gives smoother mean fields compared to the uniform time-stepping case. The present local time-stepping method is very simple to implement and requires less than $3 \%$ additional CPU time per time step for the nonreacting bluff-body calculations, which is a negligible penalty compared to the substantial reduction in the number of time steps required to reach the steady state.

\section{Acknowledgments}

This work is supported in part by Department of Energy Grant DE-FG02-90ER 14128 and in part by Grant DE-AC26-00NT40847.

\section{References}

${ }^{1}$ Turkel, E., "A Review of Preconditioning Methods for Fluid Dynamics," Applied Numerical Mathematics, Vol. 12, 1993, pp. 257-284.
${ }^{2}$ Hirsch, C., Numerical Computation of Internal and External Flows, Wiley, New York, 1994.

${ }^{3}$ Pope, S. B., "PDF Methods for Turbulent Reactive Flows," Progress in Energy and Combustion Science, Vol. 11, 1985, pp. 119-192.

${ }^{4}$ Muradoglu, M., Pope, S. B., Jenny, P., and Caughey, D. A., "A Consistent Hybrid Finite-Volume/Particle Method for the PDF Equations of Turbulent Reactive Flows,' Journal of ComputationalPhysics, Vol. 154, 1999, pp. 342 371 .

${ }^{5}$ Muradoglu, M., Pope, S. B., and Caughey, D. A., "The Hybrid Method for the PDF Equations of Turbulent Reactive Flows: Consistency Conditions and Correction Algorithms," Journal of Computational Physics, Vol. 172 2001, pp. 841-878.

${ }^{6}$ Mobus, H., Gerlinger, P., and Bruggemann, D., "Comparison of Eulerian and Lagrangian Monte Carlo PDF Methods for Turbulent Diffusion Flames," Combustion and Flame, Vol. 124, 2001, pp. 519-534.

${ }^{7}$ Dally, B. B., Fletcher, D. F., and Masri, A. R., "Measurements and Modeling of a Bluff-Body Stabilized Flame," Combustion Theory and Modelling, Vol. 2, 1998, pp. 193-219.

${ }^{8}$ Jenny, P., Muradoglu, M., Pope, S. B., and Caughey, D. A., "PDF Simulations of a Bluff-Body Stabilized Flow," Journal of Computational Physics, Vol. 169, 2001, pp. 1-23.

${ }^{9}$ Pope, S. B., "Lagrangian PDF Methods for Turbulent Flows," Annual Review of Fluid Mechanics, Vol. 26, 1994, pp. 23-63.

${ }^{10}$ Van Slooten, P. R., Jayesh, and Pope, S. B., "Advances in PDF Modeling for Inhomogeneous Turbulent Flows," Physics of Fluids, Vol. 10, 1998, pp. 246-265.

${ }^{11}$ Pope, S. B., Turbulent Flows, Cambridge Univ. Press, Cambridge, England, U.K., 2000.

${ }_{12}$ Dreeben, T. D., and Pope, S. B., "PDF/Monte Carlo Simulation of NearWall TurbulentFlows," Journal of Fluid Mechanics, Vol. 357, 1997,pp. 141166.

${ }^{13} \mathrm{Xu}$, J., and Pope, S. B., "Assessment of Numerical Accuracy of PDF/Monte Carlo Methods for Turbulent Reactive Flows," Journal of Computational Physics, Vol. 152, 1999, pp. 192-230. 